November 6, 2018 12:30 WSPC/INSTRUCTION FILE two-loop-r

\title{
TWO-LOOP CROSSOVER SCALING FUNCTIONS OF THE $O(N)$ MODEL
}

\author{
DENJOE O'CONNOR ${ }^{1, *}$, J. A. SANTIAGO ${ }^{2, \dagger}$, C. R. STEPHENS ${ }^{3, \S}$ and A. ZAMORA ${ }^{2, \ddagger}$ \\ 1 School of Theoretical Physics, Dublin Institute for Advanced Studies, 10 Burlington Road, \\ Dublin 4, Ireland \\ ${ }^{2}$ Departamento de Matemáticas Aplicadas y Sistemas, Universidad Autónoma Metropolitana - \\ Cuajimalpa, México D.F. 01120, Mexico \\ ${ }^{3}$ Instituto de Ciencias Nucleares, Universidad Nacional Autónoma de México, Apartado Postal \\ 70-543, México D.F. 04510, Mexico \\ *denjoe@stp.dias.ie \\ †jsantiago@correo.cua.uam.mx \\ $\S$ stephens@nucleares.unam.mx \\ ‡zamora@correo.cua.uam.mx \\ Received Day Month Year \\ Revised Day Month Year
}

\begin{abstract}
Using Environmentally Friendly Renormalization, we present an analytic calculation of the series for the renormalization constants that describe the equation of state for the $O(N)$ model in the whole critical region. The solution of the beta-function equation, for the running coupling to order two loops, exhibits crossover between the strong coupling fixed point, associated with the Goldstone modes, and the Wilson-Fisher fixed point. The Wilson functions $\gamma_{\lambda}, \gamma_{\varphi}$ and $\gamma_{\varphi^{2}}$, and thus the effective critical exponents associated with renormalization of the transverse vertex functions, also exhibit non-trivial crossover between these fixed points.
\end{abstract}

Keywords: Renormalization group; crossover behavior; Wilson functions.

PACS numbers: 64.60.ae, 64.60.De, 64.60.F-

\section{Introduction}

The equation of state for the $O(N)$ model remains a subject of great interest (see, for instance, Refs. 1, 2 for recent reviews). It exhibits crossover behavior between three distinct asymptotic regimes - the critical region approached along the critical isotherm, the critical region approached along the critical isochore, and, finally, the coexistence curve. For $N=1$, the longitudinal correlation length remains finite away from the critical point on the coexistence curve, while for $N>1$, the existence of Goldstone bosons leads to infrared singularities ${ }^{3 / 4}$ The problem of encapsulating these distinct scaling behaviors within one overall scaling function has been solved through an ab initio derivation from an underlying microscopic model $!^{5}$ Specifically, in that work we obtained the equation of state for the $O(N)$ model using only the 
Landau-Ginzburg-Wilson (LGW) Hamiltonian by implementing an Environmentally Friendly Renormalization (EFR) Group 6 which tracks the crossover between the fixed points that control the different asymptotic regimes.

EFR is a formalism within a general framework of perturbative renormalization and the renormalization group, specifically designed to describe crossover phenomena, where the effective degrees of freedom at different scales can be quite distinct, leading to different scaling regimes and associated exponents. To do this, a successful renormalization should track the evolving nature of the effective degrees of freedom as a function of scale and that as the latter depend on the "environment" the reparametrization chosen should also depend of it. To illustrate this, if one considered an interacting field theory in a three dimensional box of size $L$, one could renormalize the theory in an $L$ independent fashion. When one considered physics on scales $\kappa \sim L^{-1}$ one would find that the theory was perturbatively ill defined, whereas an appropriate $L$ dependent renormalization made perturbative sense. The reason for this, of course, is that the effective degrees of freedom in the system are explicitly $L$ dependent. An $L$ independent renormalization ignores this important physical fact. The only fluctuations being absorbed into the renormalized parameters in this case are $L$ independent, no matter what renormalization scale one chooses. $L$ here is the parameter which induces the crossover and therefore a good renormalization scheme should be $L$ dependent. In principle, basically any system will exhibit crossover behavior in some regime. Some pertinent examples are: systems with a bicritical point ${ }^{7}$, bulk-surface crossovers 8 and dimensional crossover ${ }^{9}$.

The advantage of this method relative to standard RG techniques is that it describes perturbatively the crossovers between any and all fixed points as opposed to the perturbative regime around one single fixed point. The disadvantage is that the Feynman diagrams that enter in the perturbative calculations are computed in the relevant environment where the finite part not just the asymptotic divergence is crucial. Applying EFR to study the crossovers inherent in the equation of state we obtain explicit functional forms that obey all required analyticity properties and require no phenomenological input, only the three Wilson functions $\gamma_{\lambda}, \gamma_{\varphi}$, and $\gamma_{\varphi^{2}}$ which are deduced from within the theory. In particular, the case $N=1$ was treated analytically in the one-loop approximation in Ref. [5. In a recent study 10 we carried out the task again in the one-loop approximation, but now to include all $N \geq 1$.

In this paper we continue the extension towards the two-loop approximation of the universal equation of state by deriving the Wilson scaling functions to this order. The main motivation for doing this is to contribute with the crossover functions required to obtain the equation of state, and to calculate the first non-trivial correction to the critical effective exponent $\eta$, which follows directly from the Wilson function $\gamma_{\varphi}$. Such a result is useful also as a checkout as no analytic form can be accessed to this order even for $N=1$. As a considerable part of the formidable task of getting the equation of state for the $O(N)$ model to order two loops, we consider it worthwhile to present the calculation in this publication. To begin with, 
in Section 2 we outline the renormalization group representation for the ab initio formulation that will be considered throughout this paper. Then, in Section 3 we obtain explicit expressions to the order of two loops for the bare vertex functions, the renormalization constants and the Wilson functions. In Section 4 we derive the two-loop order Wilson functions and solve the beta-function equation of the running coupling, whose curve exhibits the crossover between the Wilson-Fisher and strong-coupling fixed points for $N>1$. The corresponding crossovers in the Wilson functions, and therefore in the effective critical exponents, are also presented in this section. Finally, we draw concluding remarks in Section 5 .

\section{Renormalization Group Representation}

The model is described by the standard LGW Hamiltonian with $O(N)$ symmetry

$$
\mathcal{H}[\varphi]=\int \mathrm{d}^{d} x\left(\frac{1}{2} \nabla \varphi^{a} \nabla \varphi^{a}+\frac{1}{2} r(x) \varphi^{a} \varphi^{a}+\frac{\lambda_{B}}{4 !}\left(\varphi^{a} \varphi^{a}\right)^{2}\right),
$$

which describes an $N$-component scalar field $\varphi$ in a $d$-dimensional space. Here $r$ denotes the bare mass parameter, $r=r_{c}+t_{B}$, with $r_{c}$ being the value of $r$ at the critical temperature $T_{c}$ and $t_{B}=\Lambda^{2}\left(\frac{T-T_{c}}{T_{c}}\right)$, where $\Lambda$ is the microscopic scale. The value $r_{c}$ can naturally be interpreted in statistical mechanics as $r_{c} \propto T_{c}-T_{m}$, where $T_{m}$ is the critical temperature predicted by mean field theory. As is well known, an additive renormalization for $r_{c}$ is first necessary to compensate for the critical temperature shift; then a further multiplicative renormalization of $t_{B}$ is needed.

The generator of connected correlation functions, $W$, is given by

$$
W\left[H_{a}\right]=\ln Z,
$$

where $Z$ is the functional integral over the order parameter fields $\varphi^{a}$, with Hamiltonian (11) and an external source $H_{a}(x)$

$$
Z[H]=\int[d \varphi] e^{-\mathcal{H}[\varphi]+\int d^{d} x H_{a} \varphi^{a}} .
$$

The connected correlation functions are obtained by repeated functional differentiation of $W$ with respect to $t_{B}(x)$ and $H_{a}(y)$. We denote these by

$$
G_{a_{1} \ldots a_{N}}^{(N, M)}\left(x_{1}, \ldots, x_{N}, y_{1}, \ldots, y_{M}\right) .
$$

In the same fashion, the vertex functions $\Gamma_{a_{1} \ldots a_{N}}^{(N, M)}$ are obtained by functional differentiation with respect to $\bar{\varphi}(x)$ and $t(x)$ of the effective action $\Gamma[\bar{\varphi}]$, which is given as the Legendre transform

$$
\Gamma[\bar{\varphi}]=-W\left[H_{a}\right]+\int d^{d} x H_{a}(x) \bar{\varphi}^{a}(x),
$$

where $\bar{\varphi}^{a}(x)$ is the physical magnetization of the system defined as

$$
\bar{\varphi}^{a}(x)=\left.Z^{-1} \frac{\delta Z}{\delta H_{a}(x)}\right|_{H_{a}=0} .
$$


In the ordered phase two types of modes exist: those along the external field $H_{a}$ and those perpendicular to it. If we denote by $n^{a}$ to the unit vector in the direction of the external field, then by using the projectors

$$
P_{\ell}^{a b}=n^{a} n^{b}, \quad P_{t}^{a b}=\delta^{a b}-n^{a} n^{b},
$$

we can write a general vertex function as $\Gamma_{\ell \ldots \ell t \ldots t}^{(N, M)}$. When all subscripts are equal to $\ell$ or $t$, we compact them to one. For instance, $\Gamma_{t \ldots t}^{(N, M)}$ is denoted $\Gamma_{t}^{(N, M)}$. Additionally, if there are no $\varphi^{2}$ insertions (i.e. $M=0$ ), the second superscript is omitted. That is, we write $\Gamma^{(N, 0)}=\Gamma^{(N)}$.

As a consequence of the Ward identities of this model, all vertex functions can be expressed in terms of the transverse vertex functions. For instance, from the equation of state $\Gamma_{a}^{(1)}=H_{a}$ we have $\Gamma_{t}^{(1)}=0$ and $\Gamma_{\ell}^{(1)}=H$, so that use of the Ward identity $\Gamma_{\ell}^{(1)}=\Gamma_{t}^{(2)} \bar{\varphi}$ yields

$$
\Gamma_{t}^{(2)} \bar{\varphi}=H, \quad \Gamma_{t}^{(1)}=0 .
$$

Decomposing $\Gamma_{a b}^{(2)}$ produces $\Gamma_{\ell}^{(2)}, \Gamma_{t}^{(2)}$ and $\Gamma_{\ell t}^{(2)}$. Ward identities then imply

$$
\Gamma_{\ell}^{(2)}=\Gamma_{t}^{(2)}+\frac{\Gamma_{t}^{(4)}}{3} \bar{\varphi}^{2} \quad \text { and } \quad \Gamma_{\ell t}^{(2)}=0 .
$$

Analogously, one may express any vertex function in terms of the $\Gamma_{t}^{(N, M)}$. In this sense, the transverse vertex functions are the building blocks of the theory. The Wilson functions $\gamma_{i}$, to be defined shortly, can in particular be written in terms of these functions. 6

Due to the existence of large fluctuations in the critical regime, a renormalization of the microscopic bare parameters of the form

$$
\begin{aligned}
t\left(m_{t}, \kappa\right) & =Z_{\varphi^{2}}^{-1}(\kappa) t_{B}\left(m_{t}\right), \\
\lambda(\kappa) & =Z_{\lambda}(\kappa) \lambda_{B}, \\
\bar{\varphi}(\kappa) & =Z_{\varphi}^{-1 / 2}(\kappa) \bar{\varphi}_{B},
\end{aligned}
$$

must be imposed, where $\kappa$ is an arbitrary renormalization scale and $m_{t}$ is the inverse transverse correlation length. The renormalized parameters satisfy the differential equations

$$
\begin{array}{rlrl}
\kappa \frac{\mathrm{d} t(\kappa)}{\mathrm{d} \kappa}=\gamma_{\varphi^{2}}(\kappa) t(\kappa), & \text { where } & \gamma_{\varphi^{2}}(\kappa) & =-\left.\kappa \frac{\mathrm{d}}{\mathrm{d} \kappa} \ln Z_{\varphi^{2}}\right|_{c}, \\
\kappa \frac{\mathrm{d} \lambda(\kappa)}{\mathrm{d} \kappa}=\gamma_{\lambda}(\kappa) \lambda(\kappa), & & \text { where } & \gamma_{\lambda}(\kappa)=\left.\kappa \frac{\mathrm{d}}{\mathrm{d} \kappa} \ln Z_{\lambda}\right|_{c}, \\
\kappa \frac{\mathrm{d} \bar{\varphi}(\kappa)}{\mathrm{d} \kappa}=-\frac{1}{2} \gamma_{\varphi}(\kappa) \bar{\varphi}(\kappa), & \text { where } & \gamma_{\varphi}(\kappa)=\left.\kappa \frac{\mathrm{d}}{\mathrm{d} \kappa} \ln Z_{\varphi}\right|_{c},
\end{array}
$$

where on the right-hand side are the Wilson functions associated with this coordinate transformation and the derivatives are taken along an appropriately chosen 
curve in the phase diagram, which we here denote by $c$. In this paper we are interested precisely in finding the $\gamma_{i}$ as crossover scaling functions to the order of two loops.

Integration of the RG equation for any multiplicatively renormalizable $\Gamma_{t}^{(N, M)}$ yields

$$
\Gamma_{t}^{(N, M)}(t, \lambda, \bar{\varphi})=\mathrm{e}^{\int_{\kappa}^{m_{t}}\left(\frac{N}{2} \gamma_{\varphi}-M \gamma_{\varphi^{2}}\right) \frac{d x}{x}} \Gamma_{t}^{(N, M)}(t(\kappa), \lambda(\kappa), \bar{\varphi}(\kappa)) .
$$

The renormalization constants $Z_{\varphi}, Z_{\varphi^{2}}$ and $Z_{\lambda}$ are fixed by imposing the explicitly magnetization-dependent normalization conditions on the transverse correlation functions

$$
\begin{aligned}
\left.\partial_{p^{2}} \Gamma_{t}^{(2)}(p, t(\kappa, \kappa), \lambda(\kappa), \bar{\varphi}(\kappa), \kappa)\right|_{p^{2}=0} & =1, \\
\Gamma_{t}^{(2,1)}(0, t(\kappa, \kappa), \lambda(\kappa), \bar{\varphi}(\kappa), \kappa) & =1, \\
\Gamma_{t}^{(4)}(0, t(\kappa, \kappa), \lambda(\kappa), \bar{\varphi}(\kappa), \kappa) & =\lambda,
\end{aligned}
$$

while the condition

$$
\kappa^{2}=\Gamma_{t}^{(2)}(0, t(\kappa, \kappa), \lambda(\kappa), \bar{\varphi}(\kappa), \kappa)
$$

serves as a gauge fixing condition that relates the sliding renormalization scale $\kappa$ to the physical temperature $t$ and magnetization $\bar{\varphi}$. Physically, $\kappa$ is a fiducial value of the nonlinear scaling field $m_{t}$.

Besides $m_{t}$, the other nonlinear scaling field we use to parametrize our results is

$$
m_{\varphi}^{2}=\frac{1}{3} \frac{\Gamma_{t}^{(4)} \bar{\varphi}^{2}}{\left.\partial_{p^{2}} \Gamma_{t}^{(2)}\right|_{p^{2}=0}},
$$

which is RG invariant. It represents the anisotropy in the masses of the longitudinal and transverse modes and is related to the stiffness constant $\rho_{s}=\left.\bar{\varphi}^{2} \partial_{p^{2}} \Gamma_{t}^{(2)}\right|_{p^{2}=0}$ via $m_{\varphi}^{2}=\frac{1}{3} \lambda \rho_{s}$. With this renormalization prescription one may determine the Wilson scaling functions in terms of the nonlinear scaling fields $m_{t}$ and $m_{\varphi}$, as the transverse and longitudinal propagators that appear in all perturbative diagrams can be parametrized in terms of them.

\section{Perturbative Series}

\subsection{The bare correlation functions}

Within the $a b$ initio formulation we are using, there appears the difficulty of calculating Feynman diagrams that are no longer simple numbers but functions of the variables $m_{t}$ and $m_{\varphi}$ instead. In the one-loop approximation it is possible to evaluate the integrals involved analytically; however to higher orders this is no longer possible and this, numerically, complicates derivation of the equation of state. 
In this section we present a perturbative expansion of the correlation functions and show explicitly their dependence on the nonlinear scaling functions. We start from the two-loop effective action

$$
\begin{aligned}
\Gamma[\bar{\varphi}]= & \int \mathrm{d}^{d} x \frac{1}{2}\left[\bar{\varphi}^{\imath}(x)(\Delta+r) \bar{\varphi}^{\imath}(x)+\frac{\lambda}{4 !} \bar{\varphi}^{4}(x)\right] \\
& +\frac{\lambda}{4 !} \int \mathrm{d}^{d} x\left[3 G_{\ell}^{2}(x, x)+2(N-1) G_{\ell}(x, x) G_{t}(x, x)\right. \\
& \left.+\left(N^{2}-1\right) G_{t}^{2}(x, x)\right]-\frac{\lambda^{2}}{36} \int d^{d} x d^{d} y \bar{\varphi}^{\imath}(x)\left[3 G_{\ell}^{3}(x, y)\right. \\
& \left.+(N-1) G_{\ell}(x, y) G_{t}^{2}(x, y)\right] \bar{\varphi}^{\imath}(y),
\end{aligned}
$$

which embodies the physics of the system. Notice that we are implicitly working with bare quantities and to simplify the writing we will be using diagrammatic notation. We represent the longitudinal propagator by a solid line and the transverse propagator by a dotted line: $G_{\ell}^{-1}=p^{2}+r+\frac{\lambda}{2} \bar{\varphi}^{2}$ and $G_{t}^{-1}=p^{2}+r+\frac{\lambda}{6} \bar{\varphi}^{2}$ respectively. It can be observed that in the symmetric ordered phase (magnetization $\bar{\varphi}=0$ ) the two propagators are equivalent. Moreover, for models in the Ising universality class $(N=1)$ the terms mixing propagators have no contribution in the correlation function.

To obtain the two point correlation function, we take two functional derivatives of $\Gamma[\bar{\varphi}]$ respect to the order parameter $\bar{\varphi}$. After a large but otherwise direct calculation, we find the two-loop approximation for the two point correlation function in 
momenta space

$$
\begin{aligned}
& \Gamma_{\imath \jmath}^{(2)}(p)=-\frac{\lambda}{4 !}\left[6 \bigcirc+\frac{2}{3}(N-1) \bigcirc \bigcirc\right. \\
& +2(N-1) \bigcirc+\frac{2}{3}\left(N^{2}-1\right) \circlearrowright{ }^{\delta_{\imath \jmath}} \\
& +\frac{\lambda^{3}}{4 !}\left[12 \bigcirc+6 \bigcirc{ }^{2}+\frac{4}{3}(N-1) \bigcirc\right. \\
& +\frac{4}{9}(N-1) \bigcirc+4(N-1) \bigcirc \\
& +\frac{2}{9}\left(N^{2}-1\right) \circlearrowright{ }^{2}+\frac{4}{9}\left(N^{2}-1\right) \circlearrowright \bar{\varphi}^{\imath} \bar{\varphi}^{3} \\
& -\frac{\lambda^{2}}{36}\left[6 \Longleftrightarrow \delta_{\imath \jmath}-18 \lambda\left(\Longleftrightarrow+\bigodot^{0}\right) \bar{\varphi}^{\imath} \bar{\varphi}^{\jmath}-9 \lambda \bar{\varphi}^{2} \Longleftrightarrow \delta_{\imath \jmath}\right. \\
& +9 \lambda^{2} \bar{\varphi}^{2}(\Longleftrightarrow+\Longleftrightarrow 0) \bar{\varphi}^{\imath} \bar{\varphi}^{3}+9 \lambda^{2} \bar{\varphi}^{2}(\Longleftrightarrow \\
& \left.+\bigodot 0) \bar{\varphi}^{\imath} \bar{\varphi}^{\jmath}\right]-(N-1) \frac{\lambda^{2}}{36}\left[2 \longleftrightarrow \lambda_{\imath \jmath}-2 \lambda(\longleftrightarrow\right. \\
& +\Longleftrightarrow 0) \bar{\varphi}^{\imath} \bar{\varphi}^{\jmath}-\frac{2}{3} \lambda(\Longleftrightarrow 0) \bar{\varphi}^{\imath} \bar{\varphi}^{\jmath} \\
& -\lambda \bar{\varphi}^{2} \longleftrightarrow \delta_{\imath \jmath}+\lambda^{2} \bar{\varphi}^{2}(\overleftrightarrow{\longleftrightarrow}+\longleftrightarrow)^{\imath} \bar{\varphi}^{\jmath} \\
& \left.+\frac{1}{3} \lambda^{2} \bar{\varphi}^{2}(\leftrightarrow+\leftrightarrow)^{0}\right) \bar{\varphi}^{\imath} \bar{\varphi}^{3} \\
& -\frac{2}{3} \lambda \bar{\varphi}^{2} \Longleftrightarrow \delta_{\imath \jmath}-\frac{2}{3} \lambda\left(\bigodot^{0}\right) \bar{\varphi}^{\imath} \bar{\varphi}^{\jmath} \\
& +\frac{1}{3} \lambda^{2} \bar{\varphi}^{2}\left(\overleftrightarrow{\longleftrightarrow}+\bigodot^{0}+\frac{1}{3}\left(\longleftrightarrow^{0}\right)\right. \\
& \left.\left.+\frac{2}{3}(\longleftrightarrow+\longleftrightarrow 0)\right) \bar{\varphi}^{\imath} \bar{\varphi}^{\jmath}\right] \text {, }
\end{aligned}
$$

where $p$ denotes the external momenta flowing through the diagrams, the index 0 indicating evaluation of the corresponding diagram at the point $p^{2}=0$. By using the projectors (7) onto this expression one may identify both the transverse $\Gamma_{t}^{(2)}(p)$ and the longitudinal $\Gamma_{\ell}^{(2)}(p)$ two point correlation functions. The transverse two 
point vertex function is given by

$$
\begin{aligned}
& \Gamma_{t}^{(2)}(0)=t+\frac{\lambda}{6} \bar{\varphi}^{2}+\frac{\lambda}{2} \bigcirc+(N-1) \frac{\lambda}{6} \bigcirc \\
& -\frac{\lambda}{4 !}\left[6 \bigcirc \bigcirc+\frac{2}{3}(N-1) \bigcirc \bigcirc+2(N-1) \bigcirc \bigcirc\right. \\
& +\frac{2}{3}\left(N^{2}-1\right) \bigcirc \bigcirc-\frac{\lambda^{2}}{36}\left[6 \bigodot-9 \lambda \bar{\varphi}^{2} \bigcirc\right] \\
& -(N-1) \frac{\lambda^{2}}{36}\left[2 \Longleftrightarrow-\lambda \bar{\varphi}^{2}\left(\Longleftrightarrow+\frac{2}{3} \Longleftrightarrow\right)\right],
\end{aligned}
$$

where we have added the terms arising from the one-loop contribution. The derivative of this function respect to the external momenta $p$ is also required. For this we find

$$
\begin{aligned}
& \partial_{p^{2}} \Gamma_{t}^{(2)}(0)=1-\frac{\lambda^{2}}{36}\left[6 \bigotimes-9 \lambda \bar{\varphi}^{2} \bigotimes\right]
\end{aligned}
$$

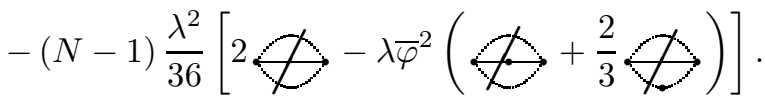

To obtain this result we have taken the derivative and then evaluated at the point $p^{2}=0$. We emphasize this fact with a diagonal line crossing the diagrams.

The four point correlation function can be calculated by taking four derivatives in Eq. (22) or alternatively by using the Ward identity (9). By using the latter we get to

$$
\begin{aligned}
& \Gamma_{t}^{(4)}(0)=\lambda-\frac{3}{2} \lambda^{2}\left(\bigodot+\frac{N-1}{9} \bigcirc\right)+\frac{\lambda^{3}}{8}[12 \bigcirc \bigcirc \\
& +6 \bigcirc 2+\frac{4}{3}(N-1) \bigcirc \bigcirc+\frac{4}{9}(N-1) \bigcirc \bigcirc \\
& +4(N-1) \bigcirc \bigcirc+\frac{2}{9}\left(N^{2}-1\right) \bigcirc 2 \\
& +\frac{4}{9}\left(N^{2}-1\right) \circlearrowright \\
& +\frac{3}{2} \lambda^{3}\left[2 \bigodot-\lambda \bar{\varphi}^{2}(\Longleftrightarrow+\bigoplus)\right] \\
& +(N-1) \frac{\lambda^{3}}{12}\left[4 \Longleftrightarrow+\frac{8}{3} \bigodot\right.
\end{aligned}
$$

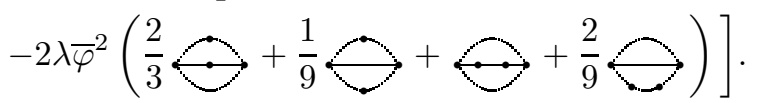

To complete this stage of the calculation we need the correlation function $\Gamma_{t}^{(2,1)}$, which can be obtained if one derivative of the function $\Gamma_{t}^{2}(p)$ respect to the param- 
eter $t$ is taken. The resulting expression is

$$
\begin{aligned}
& \Gamma_{t}^{(2,1)}=1-\frac{\lambda}{2} \bigcirc-\frac{N-1}{6} \lambda \circlearrowright+\frac{\lambda^{2}}{4 !}[6(2 \bigcirc \bigcirc \\
& \left.+\bigcirc^{2}\right)+4(N-1)\left(\frac{1}{3} \bigcirc \bigcirc+\bigcirc \bigcirc\right) \\
& +\frac{8}{3}(N-1) \bigcirc \bigcirc \\
& \left.+\frac{2}{3}\left(N^{2}-1\right)\left(2 \bigcirc+\bigcirc^{2}\right)\right] \\
& +\frac{\lambda^{2}}{4}\left[2 \bigodot-2 \lambda \bar{\varphi}^{2}(\Longleftrightarrow+\bigoplus)\right]
\end{aligned}
$$

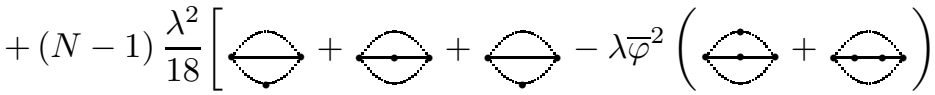

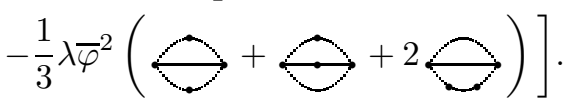

\subsection{Wave function renormalization}

We now move on to consider the wave function renormalization. To proceed, we shall use the relationships that define both scaling variables $m_{\varphi}^{2}$ and $m_{t}^{2}$ in the oneloop approximation, and then perform an inversion of these at the same order. The next step is to replace the resulting expressions into the one-loop terms within the correlation functions and then make the expansion up to two loops. By performing these expansions into the first two terms of Eq. (26) we get to the four point correlation function

$$
\begin{aligned}
& \Gamma_{t}^{(4)}(0)=\lambda_{B}-\frac{3}{2} \lambda_{B}^{2}\left(\bigodot+\frac{N-1}{9} \bigcirc\right)+\frac{\lambda_{B}^{3}}{8}\left[6 \bigcirc^{2}\right. \\
& \left.+\frac{4}{3}(N-1) \bigcirc \bigcirc+\frac{2}{9}\left(N^{2}-1\right) \bigcirc 2\right]+\frac{3}{2} \lambda_{B}^{3}[2 \bigodot \\
& \left.-\lambda_{B} \bar{\varphi}_{B}^{2}(\Longleftrightarrow+\bigodot)\right]+(N-1) \frac{\lambda_{B}^{3}}{12}\left[4 \longleftrightarrow+\frac{8}{3} \bigodot\right. \\
& \left.-2 \lambda_{B} \bar{\varphi}_{B}^{2}\left(\frac{2}{3} \Longleftrightarrow+\frac{1}{9} \Longleftrightarrow+\Longleftrightarrow+\frac{2}{9} \Longleftrightarrow\right)\right] \\
& +\frac{\lambda_{B}^{3}}{9}(N-1)[\bigodot-\bigcirc] \circlearrowright \frac{3}{2} \lambda_{B}^{4} \bar{\varphi}_{B}^{2}[\bigcirc \\
& \left.+\frac{N-1}{9} \circlearrowright\right] \bigcirc \text {. }
\end{aligned}
$$


By repeating the same procedure into the one-loop terms of $\Gamma_{t}^{(2,1)}$, one obtains

$$
\begin{aligned}
& \Gamma_{t}^{(2,1)}(0)=1-\frac{\lambda_{B}}{2}\left(\circlearrowright+\frac{N-1}{3} \bigcirc\right)+\frac{\lambda_{B}^{2}}{4 !}\left[6 \bigcirc^{2}\right. \\
& \left.+\frac{8}{3}(N-1) \bigcirc \bigcirc+\frac{2}{3}\left(N^{2}-1\right) \circlearrowright{ }^{2}\right] \\
& +\frac{\lambda_{B}^{2}}{4}\left[2 \bigodot-2 \lambda_{B} \bar{\varphi}_{B}^{2}(\Longleftrightarrow+\bigodot)\right]+(N-1) \frac{\lambda_{B}^{2}}{18}[2 \bigodot
\end{aligned}
$$

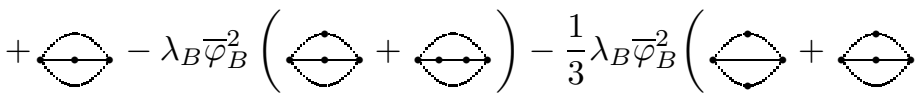

$$
\begin{aligned}
& +2 \Longleftrightarrow)]+\frac{\lambda_{B}^{2}}{9}(N-1)[\bigcirc-\bigcirc] \circlearrowright \\
& +\frac{1}{2} \lambda_{B}^{3} \bar{\varphi}_{B}^{2}\left[\bigcirc+\frac{N-1}{9} \circlearrowright\right] \bigcirc \text {. }
\end{aligned}
$$

Using these expressions we shall calculate the renormalization constants that are necessary to find the Wilson functions to the order of two loops.

\subsection{Renormalization constants}

In this section we calculate the renormalization constants or $Z$ functions. These are defined in terms of the bare correlation functions that we found in the previous section. For the function $Z_{\lambda}$ we can write

$$
\begin{aligned}
& Z_{\lambda}=1-\frac{3}{2} \lambda_{B}^{2}\left(\circlearrowright+\frac{N-1}{9} \circlearrowright\right)+\frac{\lambda_{B}^{2}}{8}\left[6 \bigcirc^{2}\right. \\
& \left.+\frac{4}{3}(N-1) \bigcirc \bigcirc+\frac{2}{9}\left(N^{2}-1\right) \bigcirc{ }^{2}\right]+\frac{3}{2} \lambda_{B}^{2}[2 \bigodot \\
& \left.-\lambda_{B} \bar{\varphi}_{B}^{2}(\Longleftrightarrow+\bigodot)\right]+(N-1) \frac{\lambda_{B}^{2}}{12}\left[\Longleftrightarrow+\frac{8}{3} \bigodot\right. \\
& \left.-2 \lambda_{B} \bar{\varphi}_{B}^{2}\left(\frac{2}{3} \Longleftrightarrow+\frac{1}{9} \Longleftrightarrow+\longmapsto+\frac{2}{9} \Longleftrightarrow\right)\right] \\
& +\frac{\lambda_{B}^{2}}{18}\left[6 \not-9 \lambda_{B} \bar{\varphi}_{B}^{2} \not\right]+(N-1) \frac{\lambda_{B}^{2}}{18}[2 \not \\
& \left.-\lambda_{B} \bar{\varphi}_{B}^{2}\left(\mathscr{C}+\frac{2}{3} \mathscr{Q}\right)\right]+\frac{\lambda_{B}^{2}}{9}(N-1)[\circlearrowright-\bigcirc] \circlearrowright \\
& +\frac{3}{2} \lambda_{B}^{3} \bar{\varphi}_{B}^{2}\left[\bigcirc+\frac{N-1}{9} \bigcirc\right] \bigcirc
\end{aligned}
$$

whereas for $Z_{\varphi}^{-1}$ the expression is

$$
\begin{aligned}
& Z_{\varphi}^{-1}=1-\frac{\lambda_{B}^{2}}{36}\left[6 \bigotimes-9 \lambda_{B} \bar{\varphi}_{B}^{2} \bigotimes\right]-(N-1) \frac{\lambda_{B}^{2}}{36}[2 \not \\
& -\lambda_{B} \bar{\varphi}_{B}^{2}\left(\not{C}+\frac{2}{3} \not\right) \text {, }
\end{aligned}
$$


and similarly for $Z_{\varphi^{2}}^{-1}$ one finds

$$
\begin{aligned}
& Z_{\varphi^{2}}^{-1}=-\frac{\lambda_{B}}{2}\left(\bigodot+\frac{N-1}{3} \bigcirc\right)+\frac{\lambda_{B}^{2}}{4 !}\left[6 \bigcirc^{2}\right. \\
& +\frac{8}{3}(N-1) \bigcirc \bigcirc+\frac{2}{3}\left(N^{2}-1\right) \circlearrowright 2 \\
& +\frac{\lambda_{B}^{2}}{4}\left[2 \bigodot-2 \lambda_{B} \bar{\varphi}_{B}^{2}(\Longleftrightarrow+\bigodot)\right] \\
& +(N-1) \frac{\lambda_{B}^{2}}{18}\left[2 \bigodot+\bigodot-\lambda_{B} \bar{\varphi}_{B}^{2}(\bigodot+\biguplus)\right. \\
& \left.-\frac{1}{3} \lambda_{B} \bar{\varphi}_{B}^{2}(\Longleftrightarrow+\Longleftrightarrow+2 \Longleftrightarrow)\right] \\
& +\frac{\lambda_{B}^{2}}{36}\left[6 \bigotimes-9 \lambda_{B} \bar{\varphi}_{B}^{2} \bigotimes\right]+(N-1) \frac{\lambda_{B}^{2}}{36}[2 \bigotimes \\
& \left.-\lambda_{B} \bar{\varphi}_{B}^{2}\left(\not{C}+\frac{2}{3} \not\right)\right]+\frac{\lambda_{B}^{2}}{9}(N-1)[\bigcirc-\bigcirc] \circlearrowright \\
& +\frac{1}{2} \lambda_{B}^{3} \bar{\varphi}_{B}^{2}\left[\bigcirc+\frac{N-1}{9} \bigcirc\right] \bigcirc
\end{aligned}
$$

Notice that we have been working with the bare coupling $\lambda_{B}$, which we now write explicitly in these expressions.

\subsection{The Wilson functions}

The Wilson functions are defined in terms of the renormalization constants $Z_{\lambda}, Z_{\varphi}$ and $Z_{\varphi^{2}}$ that we found in the previous section. From the definition of $\gamma_{\lambda}$, by using 
Eq. (30), one obtains

$$
\begin{aligned}
& \gamma_{\lambda}=-\frac{3}{2} \lambda D_{\kappa}\left(\bigodot+\frac{N-1}{9} \bigcirc\right)-\lambda^{2} D_{\kappa}\left[\frac{3}{2} \bigcirc^{2}\right. \\
& \left.+\frac{1}{3}(N-1) \bigcirc-\frac{1}{18}(N-1) \bigcirc{ }^{2}\right] \\
& +\frac{3}{2} \lambda^{2}\left[2 D_{\kappa} \bigodot-\lambda \bar{\varphi}^{2} D_{\kappa}(\Longleftrightarrow+@)\right] \\
& +(N-1) \frac{\lambda^{2}}{12}\left[4 D_{\kappa} \bigodot+\frac{8}{3} D_{\kappa} \bigodot\right. \\
& \left.-\frac{2}{3} \lambda \bar{\varphi}^{2} D_{\kappa}\left(2 \Longleftrightarrow+\frac{1}{3} \biguplus+3 \longleftrightarrow\right)\right] \\
& +\frac{\lambda^{2}}{18}\left[6 D_{\kappa} \bigotimes-9 \lambda \bar{\varphi}^{2} D_{\kappa} \bigotimes\right]
\end{aligned}
$$

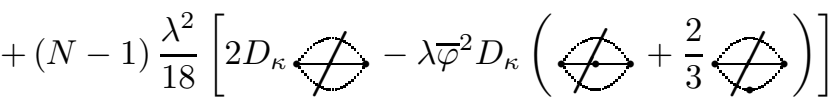

$$
\begin{aligned}
& +\frac{3}{2} \lambda^{3} \bar{\varphi}^{2} D_{\kappa}\left[\bigcirc+\frac{N-1}{9} \circlearrowright\right] \bigcirc \\
& +\frac{\lambda^{2}}{9}(N-1)\left[D_{\kappa}(\bigodot-\bigcirc) \circlearrowright-\frac{\lambda \bar{\varphi}^{2}}{3} D_{\kappa} \circlearrowright\right],
\end{aligned}
$$

where we have used $d / d \ln k:=D_{\kappa}$ to simplify the writing.

Similarly, from the definition of $\gamma_{\varphi}$ and using Eq. (31), we get

$$
\begin{aligned}
& \gamma_{\varphi}=\frac{\lambda^{2}}{36}\left[6 D_{\kappa} \bigotimes-9 \lambda \bar{\varphi}^{2} D_{\kappa} \bigotimes\right] \\
& +(N-1) \frac{\lambda^{2}}{36}\left[2 D_{\kappa} \bigotimes-\lambda \bar{\varphi}^{2} D_{\kappa}\left(\not{\bigotimes}+\frac{2}{3} \not{Q}\right)\right] \text {, }
\end{aligned}
$$


and finally from Eq. (32) we obtain for the Wilson function $\gamma_{\varphi^{2}}$,

$$
\begin{aligned}
& \gamma_{\varphi^{2}}=-\frac{\lambda}{2} D_{\kappa}\left(\circlearrowright+\frac{N-1}{3} \bigodot\right) \\
& -\lambda^{2}\left[\frac{1}{4} D_{\kappa} \bigcirc{ }^{2}+\frac{1}{18}(N-1) \circlearrowright D_{\kappa} \bigcirc\right. \\
& \left.+\frac{2}{9}(N-1) \bigcirc D_{\kappa} \bigcirc-\frac{1}{18}(N-1) D_{\kappa} \bigcirc{ }^{2}\right] \\
& +\frac{\lambda^{2}}{2}\left[D_{\kappa} \bigodot-\lambda \bar{\varphi}^{2} D_{\kappa}(\Longleftrightarrow+\bigoplus)\right] \\
& +(N-1) \frac{\lambda^{2}}{18}\left[D_{\kappa}(2 \Longleftrightarrow+\bigodot)-\lambda \bar{\varphi}^{2} D_{\kappa}(\overleftrightarrow{\bigodot}+\bigodot)\right. \\
& \left.-\frac{1}{3} \lambda \bar{\varphi}^{2} D_{\kappa}(\Longleftrightarrow+\Longleftrightarrow)\right]+\frac{\lambda^{2}}{36}\left[6 D_{\kappa} \bigotimes-9 \lambda \bar{\varphi}^{2} D_{\kappa} \bigotimes\right]
\end{aligned}
$$

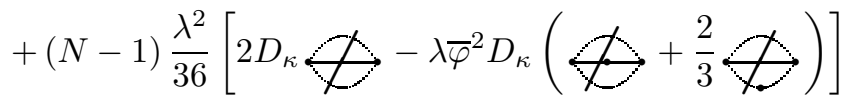

$$
\begin{aligned}
& +\frac{1}{2} \lambda^{3} \bar{\varphi}^{2} D_{\kappa}\left[\bigcirc+\frac{N-1}{9} \circlearrowright\right] \bigcirc \\
& +\frac{\lambda^{2}}{9}(N-1)\left[D_{\kappa}(\bigcirc-\bigcirc) \circlearrowright-\frac{\lambda \bar{\varphi}^{2}}{3} D_{\kappa} \circlearrowright .\right.
\end{aligned}
$$

Notice that we have applied the $D_{\kappa}$ to the renormalization constants and then replaced $\lambda_{B}$ in terms of the renormalized coupling $\lambda$. Also note that in these expressions all the diagrams are given in terms of the nonlinear scaling fields $m_{t}$ and $m_{\varphi}$ explicitly.

\section{3D Two-loop Results}

As anticipated in Section 1 we are interested in obtaining expressions for the $\gamma_{i}$ as crossover scaling functions. The natural variable is $z$, and so the next stage consists in writing the Wilson functions in terms of the nonlinear scaling fields $z=m_{t} / m_{\varphi}$. For instance, the Wilson function $\gamma_{\lambda}$ which is cubic in the coupling (see Eq. (33)) turns out a quadratic in terms of $z$. It is no difficult to express the Feynman diagrams 


$$
\begin{aligned}
& f_{1}(z)=D_{\kappa}\left(\bigodot+\frac{N-1}{9} \bigodot\right) \\
& \stackrel{3 d}{=}-\frac{1}{8 \pi^{2}}\left(\frac{N-1}{9}+\left(1+\frac{1}{z^{2}}\right)^{-3 / 2}\right), \\
& f_{2}(z)=-D_{\kappa}\left[\frac{3}{2} \bigcirc{ }^{2}+\frac{1}{3}(N-1) \bigcirc \bigcirc-\frac{1}{18}(N-1) \bigcirc{ }^{2}\right] \text {, } \\
& \stackrel{3 d}{=} \frac{1}{64 \pi^{2}}\left(-\frac{N-1}{9}+\frac{2(N-1)}{3} \frac{1+\frac{1}{2 z^{2}}}{\left(1+\frac{1}{z^{2}}\right)^{3 / 2}}+3\left(1+\frac{1}{z^{2}}\right)^{-2}\right) \text {, } \\
& f_{3}(z)=\left[2 D_{\kappa} \bigodot-\lambda \bar{\varphi}^{2} D_{\kappa}(\Longleftrightarrow+\bigotimes)\right] \text {, } \\
& \stackrel{3 d}{=}-\frac{1}{16 \pi^{3}}\left(\frac{2 \pi}{3}\left(1+\frac{1}{z^{2}}\right)^{-2}-\frac{3}{z^{2}}\left(1+\frac{1}{z^{2}}\right)^{-3}\left(\frac{\pi}{9}+\frac{1.4}{2}\right)\right),
\end{aligned}
$$

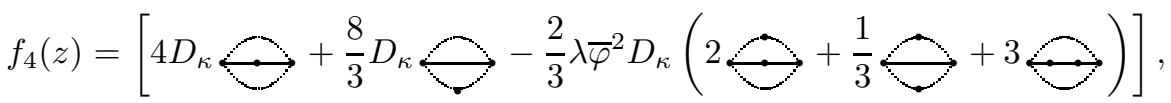

$$
\begin{aligned}
& f_{5}(z)=\left[6 D_{\kappa} \bigotimes-9 \lambda \bar{\varphi}^{2} D_{\kappa} \bigotimes\right] \text {, } \\
& \stackrel{3 d}{=} \frac{1}{72 \pi^{2}}\left(1+\frac{1}{z^{2}}\right)^{-2}-\frac{1}{24 \pi^{2}} \frac{1}{z^{2}}\left(1+\frac{1}{z^{2}}\right)^{-3} \text {, } \\
& \left.f_{6}(z)=\left[2 D_{\kappa} \bigotimes-\lambda \bar{\varphi}^{2} D_{\kappa}(\longleftrightarrow)^{+\frac{2}{3}}\right)\right] \text {, } \\
& f_{7}(z)=\lambda^{3} \bar{\varphi}^{2} D_{\kappa}\left[\bigcirc+\frac{N-1}{9} \circlearrowright\right] \bigcirc \text {, } \\
& \stackrel{3 d}{=}-\frac{3}{256 \pi^{2}} \frac{1}{z^{2}}\left(4\left(1+\frac{1}{z^{2}}\right)^{-3}+\frac{(N-1)}{9}\left(1+\frac{1}{z^{2}}\right)^{-3 / 2}\right. \\
& \left.+\frac{(N-1)}{3}\left(1+\frac{1}{z^{2}}\right)^{-5 / 2}\right) \text {, } \\
& f_{8}(z)=\frac{1}{9}\left[D_{\kappa}(\bigodot-\bigcirc)\right.
\end{aligned}
$$

the crossover scaling functions $\gamma_{\lambda}$ and $\gamma_{\varphi}$ can be written respectively as

$$
\begin{aligned}
\gamma_{\lambda}(z)= & -\frac{3}{2} f_{1} \bar{\lambda}+\left(f_{2}+\frac{3}{2} f_{3}+\frac{N-1}{12} f_{4}+\frac{1}{18} f_{5}+\frac{N-1}{18} f_{6}\right. \\
& \left.+\frac{3}{2} f_{7}+(N-1) f_{8}\right) \bar{\lambda}^{2}, \\
\gamma_{\varphi}(z)= & \left(\frac{1}{36} f_{5}+\frac{N-1}{36} f_{6}\right) \bar{\lambda}^{2} .
\end{aligned}
$$


Further, definition of the functions

$$
\begin{aligned}
& g_{1}(z)=D_{\kappa}\left(\bigodot+\frac{N-1}{3} \bigcirc\right), \\
& \stackrel{3 d}{=}-\frac{1}{8 \pi^{2}}\left(\frac{N-1}{3}+\left(1+\frac{1}{z^{2}}\right)^{-3 / 2}\right), \\
& g_{2}(z)=\left[\frac{1}{4} D_{\kappa} \bigcirc{ }^{2}+\frac{1}{18}(N-1) \bigcirc D_{\kappa} \bigcirc\right. \\
& \left.+\frac{2}{9}(N-1) \bigcirc D_{\kappa} \bigcirc-\frac{1}{18}(N-1) D_{\kappa} \bigcirc^{2}\right], \\
& \stackrel{3 d}{=}-\frac{\Gamma(1 / 2)^{2}}{(4 \pi)^{3}}\left[\frac{1}{2}\left(1+\frac{1}{z^{2}}\right)^{-2}+\frac{N-1}{18}\left(1+\frac{1}{z^{2}}\right)^{-3 / 2}\right. \\
& \left.+\frac{2(N-1)}{9}\left(1+\frac{1}{z^{2}}\right)^{-1 / 2}+\frac{N-1}{9}\right], \\
& g_{3}(z)=\left[D_{\kappa} \bigodot-\lambda \bar{\varphi}^{2} D_{\kappa}(\bigodot+\bigodot)\right] \text {, } \\
& \stackrel{3 d}{=}-\frac{1}{(4 \pi)^{3}}\left[\frac{4 \pi}{3}\left(1+\frac{1}{z^{2}}\right)^{-2}-\frac{12}{z^{2}}\left(1+\frac{1}{z^{2}}\right)^{-3}\left(\frac{\pi}{9}+\frac{1.4}{2}\right)\right], \\
& g_{4}(z)=\left[D_{\kappa}(2 \longleftrightarrow+\Longleftrightarrow)-\lambda \bar{\varphi}^{2} D_{\kappa}(\overleftrightarrow{\bigodot}+\longleftrightarrow)\right. \\
& \left.-\frac{1}{3} \lambda \bar{\varphi}^{2} D_{\kappa}(\longleftrightarrow+\biguplus)\right] \\
& g_{5}(z)=\left[D_{\kappa}(\bigodot-\bigcirc) \circlearrowright-\frac{\lambda \bar{\varphi}^{2}}{3} D_{\kappa} \Longleftrightarrow\right],
\end{aligned}
$$

allows one to write $\gamma_{\varphi^{2}}$ in the form

$$
\begin{aligned}
\gamma_{\varphi^{2}}(z)= & \frac{1}{2} f_{7}-\frac{1}{2} g_{1} \bar{\lambda}+\left(-g_{2}+\frac{1}{2} g_{3}+\frac{N-1}{18} g_{4}+\frac{1}{36} f_{5}\right. \\
& \left.+\frac{N-1}{36} f_{6}+\frac{N-1}{9} g_{5}\right) \bar{\lambda}^{2} .
\end{aligned}
$$

Note that all the crossover scaling Wilson functions are finite in the limits of small and large values of $z$. For the functions $f_{i}(z)$ and $g_{i}(z)$ for which it is not possible to obtain an exact analytical expression, we have verified using Mathematica that their behavior is finite in both asymptotic limits.

From the definition of $\gamma_{\lambda}$ and its relation to the $\beta$ function, we get the $\beta$-function equation for the dimensionless coupling $\bar{\lambda}$ :

$$
\kappa \frac{d \bar{\lambda}}{d \kappa}=-(4-d) \bar{\lambda}+\gamma_{\lambda} \bar{\lambda}
$$

As discussed in Ref. 6, one attempts to reconstruct the Wilson functions from their series to a given order in the loop expansion using some method of resummation. 
This is necessary as the direct perturbative series arising from the EFR scheme are divergent asymptotic series for any coupling strength, so that physical results can only be accessed from resummation procedures. Padé resummation is one technique, based on rational functions having the same power series expansion as the original series to the given order, that has been successfully used and is widely accepted for resumming perturbative series since the early works of Baker, 11 and so we use it here to obtain a resummed series for the beta function; i.e. right-hand side of Eq. (40). A noteworthy fact is that using different Padé approximants one may estimate errors in the resummed series. This is especially useful for higher loop computations.

There also exist more sophisticated techniques that can be used for resummation of asymptotic series, such as the Padé-Borel and conformal mapping methods. The former applies the Padé approximation to the Borel transform, whereas the latter is an improvement to the Padé-Borel method based on the mapping of a complex plane into the unit disk. More efficient techniques are based on re-expansions of the asymptotic truncated series in terms of special basis functions which are chosen to possess precisely the analytic behavior responsible for the divergence of the original series. These, and the method of variational perturbation theory, which is a systematic extension of a variational approximation to path integrals, require information on the behavior of the series, so they are suitable for higher order expansions (see Ref. 12 for a review of these methods).

For our two-loop calculations, we use for simplicity the Padé approximant. That is, we solve numerically the [2/1] Padé-resummed differential equation arising from Eq. (40). The solutions we find are shown in Fig. 1, There, one may see the coupling

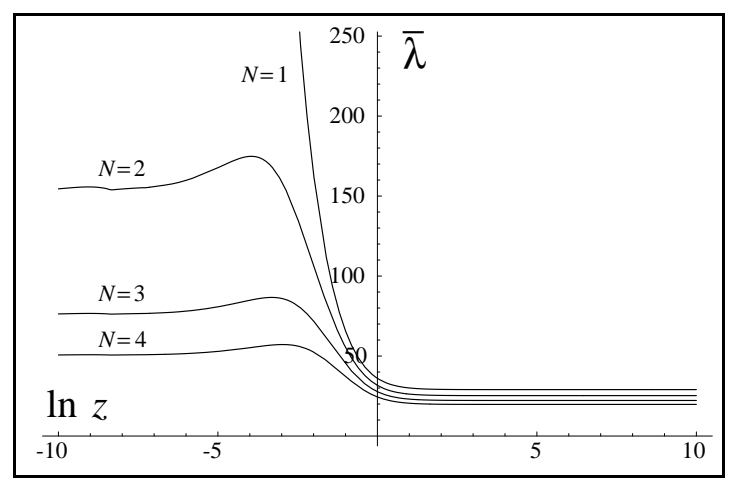

Fig. 1. The Padé-resummed coupling $\bar{\lambda}(z)$, for several values of $N$. Note the crossover to the strong fixed point for $z \rightarrow 0$.

parameter interpolating continually between the fixed points of the model. The presence of the Wilson-Fisher fixed point $\bar{\lambda}^{*}$ can be observed for large numerical values of $z$ and, for $N>1$, the fixed point associated with the coexistence curve in the limit $z \rightarrow 0$. Notice the relative decrease of the maximum, on the left of the 
curve, as $N$ increases. More precise values in the asymptotic regimes, obtained from higher order approximations that introduce external numerical data, are known in the literature. This leads one to expect the same qualitative behavior of the curve to higher orders in the loop approximation. For completeness, in Table 1 we present the asymptotic values for the coupling in the limit $z \rightarrow 0$ and in Table 2 the values corresponding to the Wilson-Fisher fixed point. However, as these values come from

$\begin{aligned} & \text { Table } \\
& \text { of the 3D coupling and Wilson }\end{aligned}$
functions in the limit $z \rightarrow 0$.
\begin{tabular}{lrcccc} 
functic \\
\hline$N$ & $\bar{\lambda}$ & $\gamma_{\lambda}$ & $\gamma_{\varphi}$ & $\gamma_{\varphi^{2}}$ \\
\hline 1 & $\infty$ & 0 & 0 & 0 \\
2 & 154.4 & 1 & 0 & 1 \\
3 & 76.6 & 1 & 0 & 1 \\
4 & 50.6 & 1 & 0 & 1 \\
\hline
\end{tabular}

Table 2. Asymptotic values of the 3D coupling, Wilson functions and critical exponents at the WF critical point.

\begin{tabular}{ccccccccc}
\hline$N$ & $\bar{\lambda}^{*}$ & $\gamma_{\lambda}^{*}$ & $\gamma_{\varphi}^{*}$ & $\gamma_{\varphi^{2}}^{*}$ & $\beta$ & $\delta$ & $\nu$ & $\gamma$ \\
\hline 1 & 29.01 & 1 & 0.033 & 0.43 & 0.33 & 4.8 & 0.64 & 1.26 \\
2 & 25.26 & 1 & 0.033 & 0.52 & 0.35 & 4.8 & 0.68 & 1.33 \\
3 & 22.23 & 1 & 0.032 & 0.59 & 0.37 & 4.8 & 0.71 & 1.39 \\
4 & 19.76 & 1 & 0.031 & 0.64 & 0.38 & 4.8 & 0.73 & 1.45 \\
\hline
\end{tabular}

a two-loop calculation, no greater precision is expected. What is worthwhile noticing is the fact that, within our ab initio calculation and without external input data, we capture the crossover-function character of the running coupling interpolating from one fixed point to the other. By substituting the numerical solution for the coupling into the Padé-resummed $\gamma_{\lambda}(z)$ function, we obtain the behavior of the Wilson functions showing the continuum crossover between the Wilson-Fisher fixed point and the fixed point associated with the coexistence curve.

The crossover scaling function $\gamma_{\lambda}(z)$ in Fig. 2 provides information on the effective dimension of the system 6 . Given that the dimension employed to evaluate the expressions is $d=3$, asymptotically the value is 1 , as expected. Nevertheless, it is of theoretical and experimental interest the local minimum values of this function for $N>1$. For $N=1$, the limit $\gamma_{\lambda} \rightarrow 0$ shows mean field behavior as the fluctuations are suppressed. On the other hand, the crossover scaling function $\gamma_{\varphi}(z)$ directly corresponds to the effective exponent $\eta$, see Fig. 3. As one would expect, in the Wilson-Fisher fixed point, this takes values that approximate those known from higher order calculations. We stress once again that, in the two-loop order approximation that we consider in this work, the contribution comes from the $a b$ 


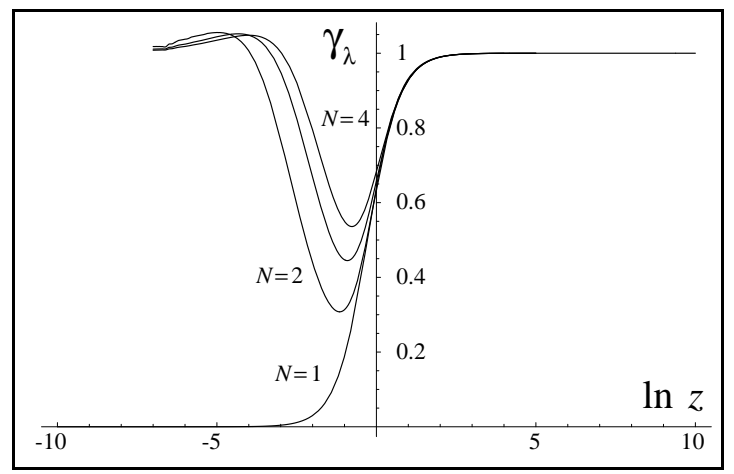

Fig. 2. The Wilson function $\gamma_{\lambda}(z)$ showing that $\gamma_{\lambda} \rightarrow 1$ in the limits $z \rightarrow \infty$ and $z \rightarrow 0$, except for $N=1$.

initio calculation and no attempt has been made at improving numerical values using external data. The numerical values that we obtain are, nevertheless, provided in table 2. Once more, in the limit $z \rightarrow 0$ the fluctuations are suppressed and this can be observed in the zero value of this anomalous exponent. Finally, the crossover

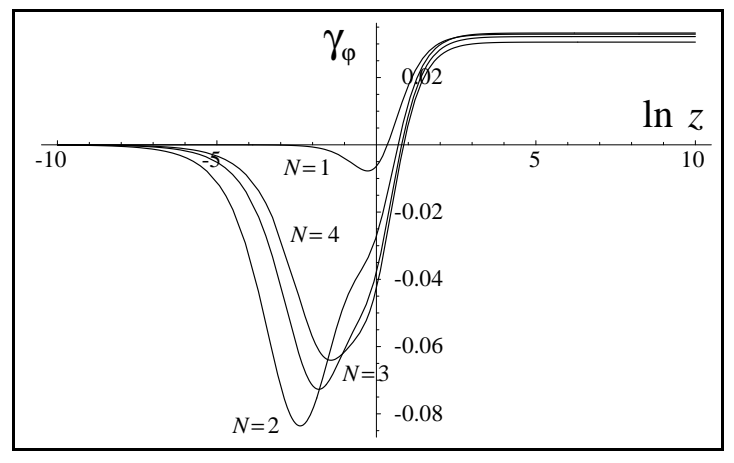

Fig. 3. The Wilson function $\gamma_{\varphi}(z)$.

scaling function $\gamma_{\varphi^{2}}$ in Fig. 4 also shows non-trivial crossover. In the asymptotic limit $z \rightarrow \infty$, this function is related to the critical exponent $\nu$. It can be observed that the curves $\gamma_{\varphi^{2}}(z)$, for $N>1$, tend to follow the trajectory described by the Ising model in the limit $z \rightarrow 0$, but in the end they separate from it. Again in this case, just as a reference, the asymptotic values of this function and for completeness the values of the critical exponents have also been included in both tables.

\section{Conclusions}

By using EFR it is possible, from an ab initio calculation, to derive a parametric form for the equation of state of the $O(N)$ model that has all desired analiticity 


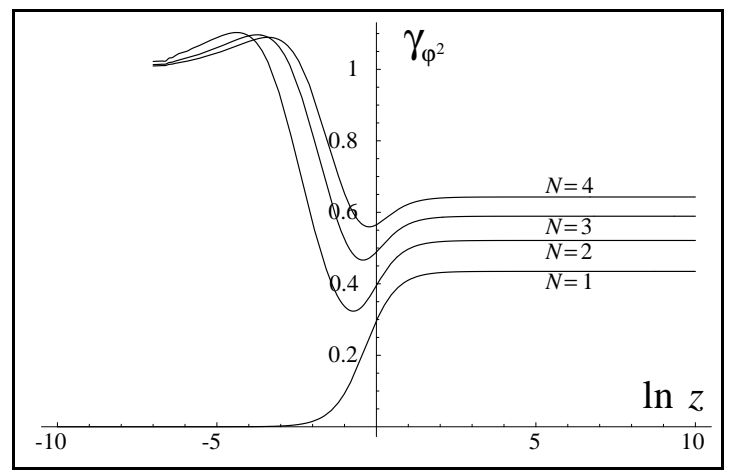

Fig. 4. The Wilson function $\gamma_{\varphi^{2}}(z)$. Notice the mean field behavior of the Ising model, $N=1$, in the limit $z \rightarrow 0$ whereas $\gamma_{\varphi^{2}} \rightarrow 1$ for $N>1$.

properties in the three distinct asymptotic regimes. To order one loop it is even possible to obtain an analytic expression for $N=1$ in dimension three. We see that the fundamental building blocks for calculations are the Wilson functions. However, despite the fact that they play a privileged role they enter into expressions for physical quantities in a non-trivial way, such as in integrals. In the case where the Wilson functions must be computed numerically in the first place this involves numerical subtleties that are not present in standard calculations, such as perturbation expansions in $\varepsilon$ or $1 / N$ for problems where, for instance, the upper critical dimension or the symmetry of the order parameter change, respectively. In reality this is to be expected, calculating a crossover scaling function is much more complicated than calculating an exponent.

From the EFR formalism it would seem that once the Wilson functions are calculated at a given order, the derivation of the equation of state at the same order should be somehow straightforward. However, the Feynman diagrams appearing in the Wilson functions to order two loops are crossover functions themselves and the difficulty precisely resides in systematically sum diagrams whose divergences do cancel. This is a problem we are currently sorting out. In this paper we have performed explicit two-loop order calculations of the transverse correlation functions, the renormalization constants and the Wilson functions. By solving numerically the beta-function equation, we have captured the crossover between the critical fixed point and the fixed point associated with the coexistence curve. The Wilson functions also show the crossover. In the limit $z \rightarrow \infty$, the Wilson-Fisher fixed point is approached and $\gamma_{i} \rightarrow \gamma_{i}^{W F}$ with $\gamma_{\lambda}=1$ for $d=3$. In contrast, in the limit $z \rightarrow 0$ the strong-coupling fixed point is approached and $\gamma_{i} \rightarrow \gamma_{i}^{S C}$. For $N>1$ the Goldstone bosons dominate and $\gamma_{\lambda}=\gamma_{\varphi^{2}}=1$. For $N=1$ however, this fixed point is mean field like as fluctuations are suppressed and $\gamma_{i} \rightarrow 0$. 


\section{Acknowledgments}

JAS would like to thank Rocío Mondragón and Claudio Santiago for support and motivation during the several stages of this work.

\section{Appendix A. Derivatives of Feynman Integrals}

We summarize here expressions for some of the diagrams appearing in the numerical functions $f_{i}(z)$ and $g_{i}(z)$. In terms of the functions

$$
\begin{aligned}
f(x, y) & =x(1-x)(1-y)+y, \\
g(x, y, z) & =f(x, y)+y(1-x) z^{-2},
\end{aligned}
$$

we can write

$$
\begin{aligned}
& D_{\kappa} \bigodot=A_{d} \int_{0}^{1} \int_{0}^{1} d x d y \frac{x^{1-d / 2}(y(1-x))^{2-d / 2} f(x, y)}{g(x, y, z)^{5-d}} \\
& D_{\kappa} \bigodot=A_{d} \int_{0}^{1} \int_{0}^{1} d x d y \frac{(x(1-x))^{2-d / 2}(1-y) y^{1-d / 2} f(x, y)}{g(x, y, z)^{5-d}}
\end{aligned}
$$

where $A_{d}=-\frac{2(4-d) \Gamma(4-d)}{(4 \pi)^{d}} \frac{1}{\kappa^{2(4-d)}}$. Analogously, we have

$$
\begin{aligned}
& D_{\kappa} \bigodot=B_{d} \int_{0}^{1} \int_{0}^{1} d x d y \frac{y^{3-d / 2}(x(1-x))^{2-d / 2} f(x, y)}{g(x, y, z)^{6-d}}, \\
& D_{\kappa} \bigodot=B_{d} \int_{0}^{1} \int_{0}^{1} d x d y \frac{(y(1-x))^{2-d / 2} x^{3-d / 2}(1-y) f(x, y)}{g(x, y, z)^{6-d}}, \\
& D_{\kappa} \Longleftrightarrow=\frac{B_{d}}{2} \int_{0}^{1} \int_{0}^{1} d x d y \frac{(y(1-x))^{3-d / 2} x^{1-d / 2} f(x, y)}{g(x, y, z)^{6-d}},
\end{aligned}
$$

where $B_{d}=-\frac{2(5-d) \Gamma(5-d)}{(4 \pi)^{d}} \frac{1}{\kappa^{2(5-d)}}$. Finally for the diagrams with the diagonal line crossing them, we find

$$
\begin{aligned}
& D_{\kappa} \not 2=-A_{d} \int_{0}^{1} \int_{0}^{1} d x d y \frac{y^{2-d / 2}(1-y)(x(1-x))^{2-d / 2} f(x, y)}{g(x, y, z)^{5-d}}, \\
& D_{\kappa} \npreceq=-B_{d} \int_{0}^{1} \int_{0}^{1} d x d y \frac{x^{1-d / 2}(y(1-x))^{3-d / 2}(1-y) f(x, y)}{g(x, y, z)^{6-d}} \text {, }
\end{aligned}
$$

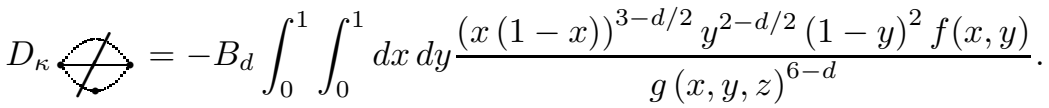

\section{References}

1. J. Zinn-Justin, Phys. Rep. 344, 159 (2001).

2. A. Pelissetto and E. Vicari, Phys. Rep. 368, 549 (2002).

3. I. D. Lawrie, J. Phys. A: Math. Gen. 14, 2489 (1981).

4. L. Schäfer and H. Horner, Z. Phys. B 29, 251 (1978). 
5. D. O'Connor, J. A. Santiago and C. R. Stephens, J. Phys. A: Math. Theor. 40, 901 (2007).

6. D. O'Connor and C. R. Stephens, Phys. Rep. 363, 425 (2002).

D. O'Connor and C. R. Stephens, Int. J. Mod. Phys. A 9, 2805 (1994).

[Erratum-ibid. A 9, 5851 (1994).]

D. O'Connor and C. R. Stephens, Nucl. Phys. B 360, 297 (1991).

7. M. E. Fisher and D. R. Nelson, Phys. Rev. Lett. 32, 1350 (1974).

8. K. Binder, in Phase Transitions and Critical Phenomena, Vol. 5B, edited by C. Domb and M. S. Green (Academic Press, New York, 1976).

9. M. N. Barber, in Phase Transitions and Critical Phenomena, Vol. 8, edited by C. Domb and J. L. Lebowitz (Academic Press, London, 1983).

10. D. O'Connor, J. A. Santiago and C. R. Stephens, J. Phys. A: Math. Theor. 42, 045003 (2009).

11. G. A. Baker, Phys. Rev. 124, 768 (1961).

G. A. Baker and J. L. Gammel, J. Math. Anal. Appl. 2, 21 (1961).

12. H. Kleinert and V. Schulte-Frohlinde, Critical Properties of $\phi^{4}$-Theories (World Scientific, Singapore, 2001), and references therein. 